\title{
SNS INJECTION SIMULATIONS WITH SPACE CHARGE*
}

\author{
J. Galambos, S. Danilov, D. Jeon, J. Holmes, D. Olsen, ORNL, Oak Ridge, TN
}

\section{Abstract}

Injection scenarios are investigated for the Spallation Neutron Source (SNS) accumulator ring using a new computer model that includes transverse space charge effects. Transverse halo growth is compared for a variety of painting schemes, lattice tunes, and lattice types. Of these, the lattice tune has the strongest impact on the halo growth.

\section{INTRODUCTION}

The SNS accumulator ring will have a high intensity ( $\left.10^{14}\right)$ low energy $(1 \mathrm{GeV})$ beam and required uncontrolled particle loss rates of $\sim$ one part in $10^{4}$. For these beam parameters, space charge effects could drive beam halo growth, which would lead to violation of the stringent loss criterion. In this paper we investigate the transverse halo growth due to space charge using a newly developed simulation tool, ORBIT [1]. We examine a variety of injection schemes, the effect of the lattice tune, and also the impact of using a FODO or doublet lattice. Painting schemes are examined for (1) directly correlated horizontal (X) - vertical (Y) closed orbit bumps, (2) anti-correlated horizontal - vertical bumps, and (3) horizontal closed orbit bump (fixed vertical smoke ring). Next we examine a wide range of lattice tunes for a fixed injection scheme. The tune is found to have a strong impact on halo growth. Finally we compare halo growth in FODO and doublet lattices with the same tunes and the same injection scenario. Neither lattice type is consistently superior regarding halo production.

The space charge model in ORBIT uses a PIC calculation with an FFT method to calculate the force [2]. For cases shown here we use 480 transverse spacecharge kicks/turn (or about 60 per betatron oscillation), 50,000 macro-particles, and a 64x64 PIC grid. We inject particles with emittance $<120 \pi$-mm-mrad, and we define halo as particles with emittance $>180 \pi$-mm$\mathrm{mrad}$, the acceptance of the SNS primary collimators. All cases are for injection of $2 \times 10^{14}$ particles. These results do not include lattice errors or wall impedance effects.

\section{PAINTING SCHEMES}

The SNS ring design allows both horizontal and vertical closed orbit bumps at the foil, providing flexibility in the possible painting schemes. Three primary painting strategies are: (1) inject correlated horizontal and vertical distributions; (2) inject anti-correlated horizontal and vertical distributions; and (3) inject a painted horizontal or vertical smoke-ring distribution. The first scheme has the advantage of pulling the painted distribution away from the foil in both directions, resulting in the fewest foil traversals. At completion, the anti-correlated painting scheme yields a distribution roughly similar to a K-V distribution. However, in this scheme one direction is pulled toward the foil during injection, resulting in higher foil traversals, and a larger aperture is required than in the other two cases. The smoke-ring scheme is the simplest, as only one bump direction is employed.

Table 1. Results of different painting scenarios.

\begin{tabular}{|l|l|l|l|}
\hline <Foil Hits> & $\begin{array}{c}\varepsilon_{\mathrm{RMS}}(\pi- \\
\mathrm{mm}-\mathrm{mrad}\end{array}$ & $\begin{array}{c}\text { Beam \% with } \\
\varepsilon_{(\mathrm{X} / \mathrm{Y})}>180 \\
(\pi-\mathrm{mm}-\mathrm{mrad})\end{array}$ & $\begin{array}{c}\text { Max. tune } \\
\text { spread } \\
(\mathrm{X} / \mathrm{Y})\end{array}$ \\
\hline \multicolumn{2}{|l|}{ Correlated painting } & & \\
\hline 2.7 & $26 / 27$ & 0.004 & $0.20 / 0.21$ \\
\hline \multicolumn{2}{|l|}{ Anti-correlated painting } & & $0.20 / 0.30$ \\
\hline 5.8 & $24 / 16$ & 0.25 & \\
\hline \multicolumn{2}{|l|}{ Vertical smoke ring painting } & $0.16 / 0.23$ \\
\hline 3.7 & $30 / 21$ & 0.038 & \\
\hline
\end{tabular}

Table 1 shows results of the three painting options. In each of these cases the closed orbit trajectory was optimized during injection painting to minimize the amount of halo produced and the tune shift. All cases use the nominal SNS FODO cell lattice with tunes of $v_{x}=$ 5.82 and $v_{y}=5.90$, which result in little beam halo. In the anti-correlated painting scheme particles are injected initially with a large emittance in one direction and a small emittance in the other direction. We chose to inject a large vertical and small horizontal emittance at the outset. This means that particles with large vertical emittance spend more time in the beam during injection, compared to the correlated case in which painting proceeds from smallest to largest emittance in both directions. In the correlated case, any emittance growth in the existing beam is continually painted over during the injection. This is one reason why the correlated painting case develops little halo. Both the smoke-ring and anti-correlated injection schemes have been optimized to reduce halo production by injecting most

* SNS research is sponsored by the Division of Materials Science, U.S. DOE, under contract number DE-AC05-960R22464 with LMER Corp. for ORNL. 
particles a smaller emittance than the final target value. However, this results in small RMS emittances and large tune spreads, which may be troublesome in the presence of magnet errors.

Figure 1 shows the real space distributions for the final correlated and anti-correlated painting schemes. The rectangular shape for the correlated painting scheme occurs because the particles with the largest horizontal actions $\mathrm{J}_{\mathrm{x}}$ also have the largest vertical actions $\mathrm{J}_{\mathrm{y}}$, and the elliptical shape for the anti-correlated scheme is that expected for a $\mathrm{K}-\mathrm{V}$ distribution. Figure 2 shows the vertical phase at the end of injection, with and without transverse space charge effects, for the vertical smoke ring case. Space charge forces spread and fill the distribution dramatically, This behavior has been observed experimentally [3].

Figure 1. Real space profiles for correlated painting,:

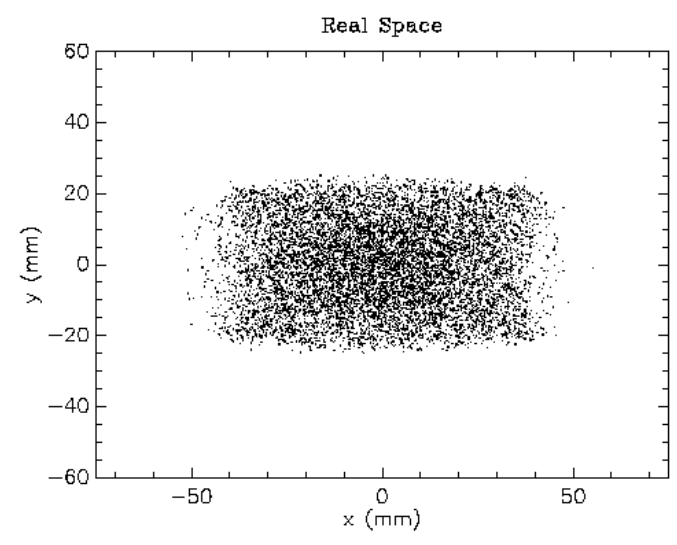

and for anti-correlated painting:

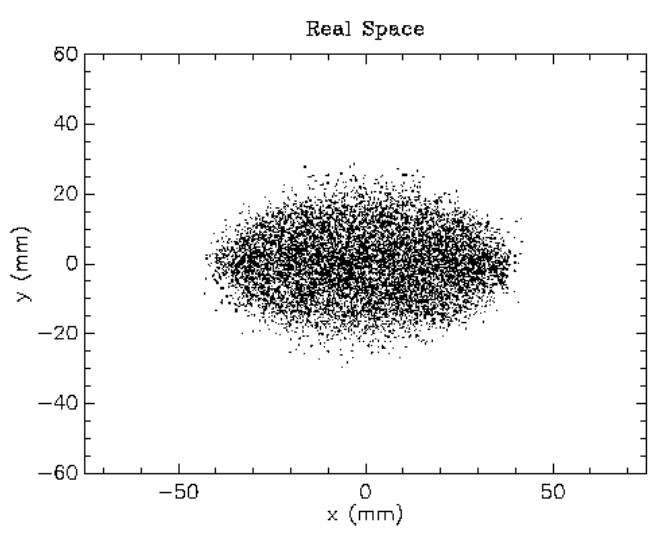

\section{OPERATIONAL TUNE}

Space-charge-driven resonances have been shown to result in rms beam mismatch that leads to halo growth [4]. In this section we survey the impact of varying the bare tune in the SNS lattice for a fixed injection painting scheme. We use a correlated $\mathrm{X}-\mathrm{Y}$ painting scheme parameterized to minimize halo production for the nominal tunes of $v_{x}=5.82$ and $v_{y}=5.80$. Figure 3 plots the resulting fraction of the beam with emittance $>180$ $\pi$-mm-mrad (in either $\mathrm{X}$ or $\mathrm{Y}$ ) vs. the bare vertical tune. For each of these cases, the horizontal tune is held fixed, and the dispersion in the ring straight sections is maintained at a level comparable to the baseline $(<0.4$ m). As seen in Fig. 3, the amount of generated halo depends strongly on the operating tune. There are large amounts of halo at tunes flanking integer values, and there are also regions of very little halo generation, for example just below the integer tunes at $v_{\mathrm{y}}=5.9$ and $v_{\mathrm{y}}=$ 4.85. The peaks and minima in this figure have not been investigated in detail, but is apparent that the choice of tune is important.

Figure 2. Vertical phase space distribution for smoke ring injection, calculated with transverse space charge:

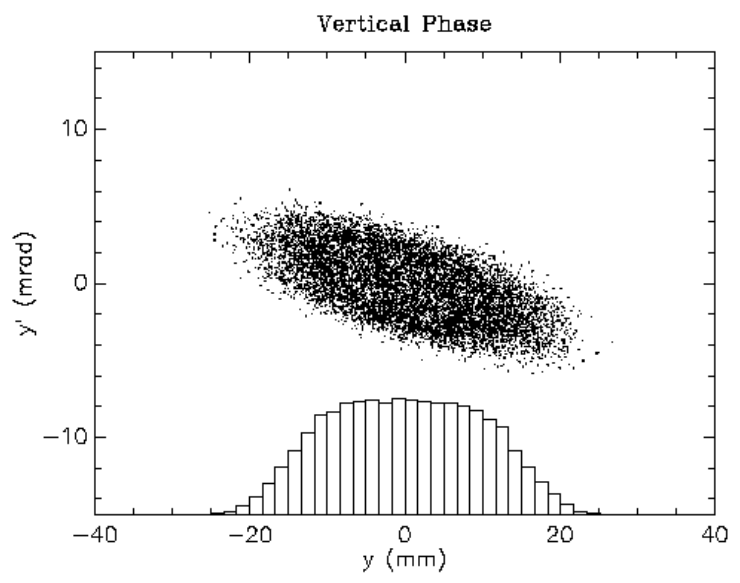

and without transverse space charge.

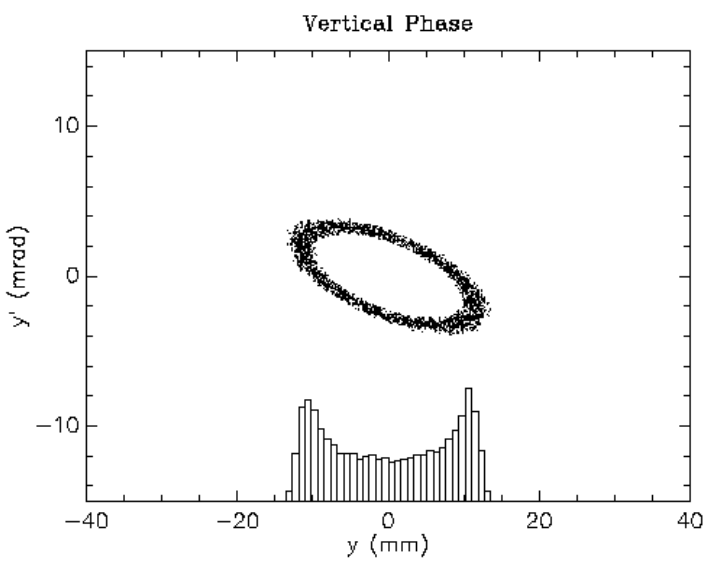

The vertical beam profiles at the end of injection for three of the cases shown in Fig. 3 are plotted in Figure 4. Even though the painting scheme is the same for all three cases, the final profile shape is affected by the lattice tune through the influence of space charge. 
Figure 3. Halo generation as a function of the lattice bare vertical tune.

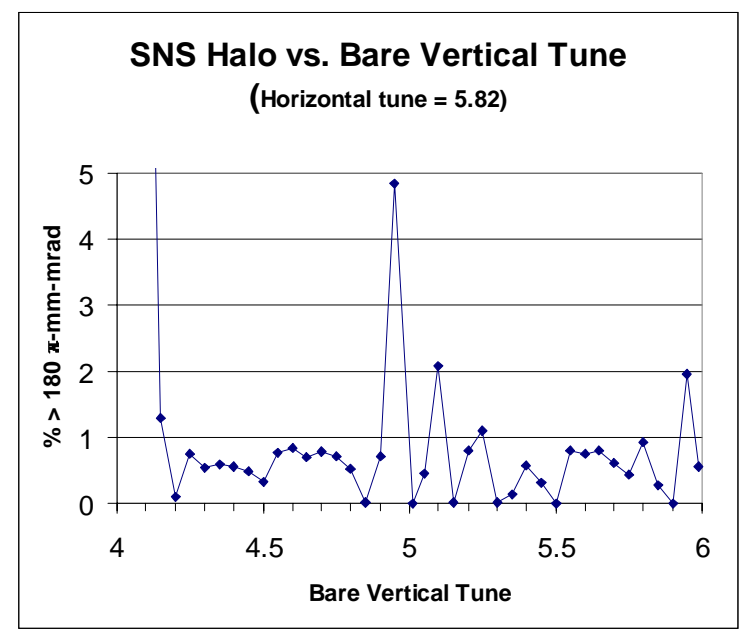

Figure 4: Final vertical beam profiles for three of the tunes shown in Figure 3.

\section{SNS Vertical Profiles}

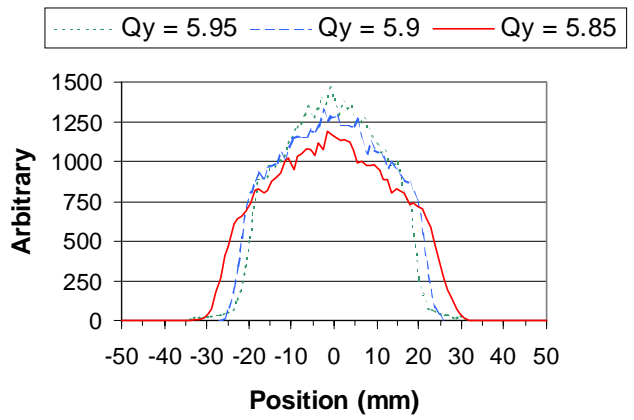

\section{LATTICE TYPE}

The baseline SNS lattice is a FODO lattice having four super-periods. We use a doublet with similar parameters (see Table 2) to test the sensitivity of the halo generation to the lattice type. The doublet lattice used here does not include the fixed injection chicane that is in the nominal SNS lattice. Figure 4 shows the fraction of the beam with emittance $>180 \pi$-mm-mrad at the end of injection for (1) the nominal SNS FODO lattice, (2) the SNS FODO lattice without the injection chicane, and (3) the doublet lattice. There is little difference between the SNS FODO lattice with and without the injection chicane. Both the doublet and FODO lattices show a similar trend of halo vs. tune, with minimum halo generation for a tune of 5.90. The doublet case has more halo for $v_{x}=5.85$, and less halo for $v_{x}=5.95$.

\section{SUMMARY}

Injection scenarios have been investigated for the SNS, with particular emphasis on minimizing the spacecharge-induced halo growth. Halo levels for correlated $\mathrm{X}-\mathrm{Y}$ painting are smaller than anti-correlated $\mathrm{X}-\mathrm{Y}$, or vertical smoke ring painting, The correlated painting scheme also results in the fewest foil traversals, and the smallest tune spread. A broad survey of the effect lattice operational tune shows a strong impact on beam halo production. Finally, we see no systematic difference in halo production between comparable doublet and FODO lattices with similar tunes and injection scenarios.

Table 2: Parameters of lattices used in halo calculation comparison.

\begin{tabular}{|l|c|c|}
\hline & $\frac{F O D O}{220.7}$ & $\frac{\text { Doublet }}{220.7}$ \\
Length (m) & 4 & 4 \\
Super-periods & $19.1 / 19.5$ & $16.2 / 16.9$ \\
$\beta_{x y} \max (\mathrm{m})$ & 4.14 & 3.80 \\
Dispersion - $\max (\mathrm{m})$ & 48 & 48 \\
\# quads & 32 & 32 \\
\# dipoles & 5.29 & 10.1 \\
Max. straight (m) & 0.5 & 0.5 \\
Quad lengths (m) & 5.29 & 1.0 \\
Quad separation (m) & \\
\hline
\end{tabular}

Figure 5: Halo generation for similar injection in a FODO and a Doublet lattice.

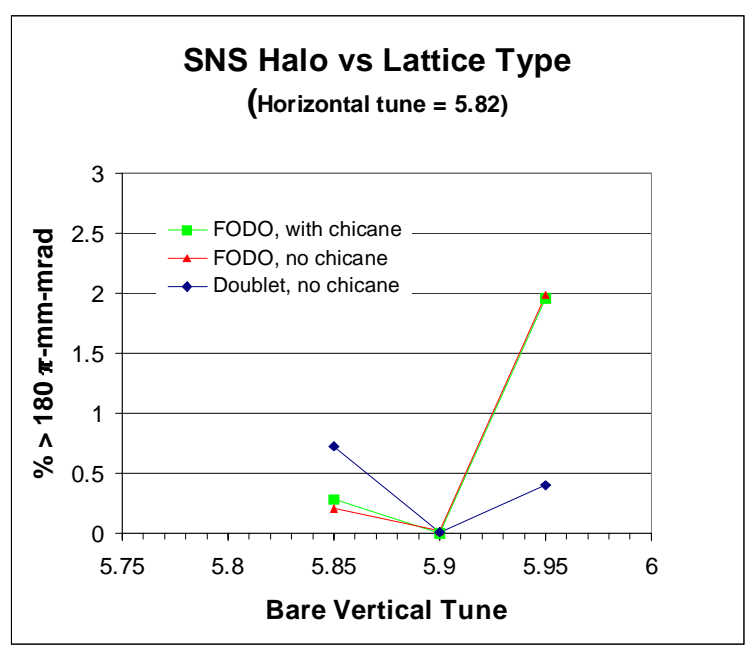

\section{REFERENCES}

1. J. Galambos, V. Danilov, D. Jeon, J. Holmes, D. Olsen, J. Beebe-Wang, A. Luccio "ORBIT - A Ring Injection Code with Space Charge, these proceedings.

2. J.A. Holmes, J.D. Galambos, D-O Jeon, D.K. Olsen, M. Blaskiewicz, A.U. Luccio, and J.J. Beebe-Wang, "Dynamic Space Charge Calculations for High Intensity Beams in Rings", presented at the International Computational Accelerator Physics Conference, Monterey, CA, September 1998.

3. J. Galambos, S. Danilov, D. Jeon. J.A. Holmes, D.K. Olsen, F. Neri, M. Plum, SNS/ORNL/AP Tech. Note 009.

4. D. Jeon, J. A. Holmes, S. Danilov, J. Galambos, and D.K. Olsen, submitted to Phys. Rev. Lett. (1999). 